\title{
Neurofeedback training with a motor imagery-based BCI: neurocognitive improvements and EEG changes in the elderly
}

Javier Gomez-Pilar ${ }^{1 *}$, Rebeca Corralejo ${ }^{1}$, Luis F. Nicolas-Alonso ${ }^{1}$, Daniel Álvarez ${ }^{1}$, Roberto Hornero ${ }^{1}$

1 Biomedical Engineering Group, E.T.S.I. de Telecomunicación, Universidad de Valladolid, Paseo Belén 15, 47011, Valladolid, Spain

* Corresponding Author:

Javier Gomez-Pilar,

e-mail: javier.gomez@gib.tel.uva.es

tel: +34983423000 ext. 4713

The total number of words of the manuscript, including entire text from title page to figure legends: 7082

The number of words of the abstract: 200

The number of figures: 6

The number of tables: 1 


\begin{abstract}
Neurofeedback training (NFT) has shown to be promising and useful to rehabilitate cognitive functions. Recently, brain-computer interfaces (BCIs) were used to restore brain plasticity by inducing brain activity with a NFT. In our study, we hypothesized that a NFT with a motor imagery-based BCI (MI-BCI) could enhance cognitive functions related to aging effects. To assess the effectiveness of our MI-BCI application, 63 subjects (older than 60 years) were recruited. This novel application was used by 31 subjects (NFT group). Their Luria neuropsychological test scores were compared with the remaining 32 subjects, who did not perform NFT (control group). Electroencephalogram (EEG) changes measured by relative power $(R P)$ endorsed cognitive potential findings under study: visuospatial, oral language, memory, intellectual and attention functions. Three frequency bands were selected to assess cognitive changes: 12,18 , and $21 \mathrm{~Hz}$ (bandwidth $3 \mathrm{~Hz}$ ). Significant increases $(p<0.01)$ in the $R P$ of these frequency bands were found. Moreover, results from cognitive tests showed significant improvements $(p<0.01)$ in four cognitive functions after performing five NFT sessions: visuospatial, oral language, memory, and intellectual. This established evidence in the association between NFT performed by a MI-BCI and enhanced cognitive performance. Therefore, it could be a novel approach to help elderly people.
\end{abstract}

\title{
Keywords
}

Brain Computer Interface (BCI), Neurofeedback Training (NFT), Electroencephalogram (EEG), Luria Adult Neuropsychological Diagnosis (Luria-AND), Elderly People 


\section{Introduction}

Advances in signal processing and computing capabilities have enabled people with different disabilities to use brain computer interfaces (BCIs) for communication or the control of devices without the involvement of peripheral nerves and muscles [44]. Electroencephalography (EEG) is the most commonly used method for monitoring brain activity in BCI systems because it is a noninvasive technique that requires relatively simple and inexpensive equipment [45].

By means of motor imagery strategies, different tasks for the activation or deactivation of specific brain signals can be proposed. Motor imagery-based BCI (MI-BCI) applications translate brain activity during the motor imagery tasks into control commands. These endogenous BCI systems depend on the user's ability to control the amplitude in a specific frequency band of the EEG recorded over a particular cortical area [45]. MI-BCI is based on the generation of event-related desynchronization (ERD) and event-related synchronization (ERS) in alpha (8-13 Hz) and beta (13-30 Hz) frequency bands of the EEG [20, 33]. These events are related to sensorimotor rhythms (SMR). The SMR show a characteristic ERD and subsequent rebound, or ERS, after motor imagery task [28]. ERD in hand movement is more prominent over the contralateral sensorimotor areas [28]. These spectral changes can be used to provide feedback by a MI-BCI system, and, at the same time, to activate endogenous EEG activity in alpha and beta frequency bands. Since EEG-alpha and EEG-beta band activity are associated with different cognitive and motor functions [3, 15, 20, 31, 32], BCIs have become not only a tool to assist people with disabilities, but also a way to rehabilitate or restore motor or cognitive functions. Brain plasticity can be restored into a more normal brain function by means of inducing brain activity [13]. In this regard, neurofeedback training (NFT) has been shown to be an appropriate way to control one's own brain activity [47]. In fact, NFT can lead to neuroplastic changes occurring after NFT [34] and microstructural changes in white and grey matter [18]. In previous studies, NFT has been used to treat different diseases such as AttentionDeficit/Hyperactivity Disorder (ADHD) [2, 17], autism [10], epilepsy [37], or Traumatic Brain Injury (TBI) [38], among others. Furthermore, there are some reports indicating that NFT might be used to increase cognitive performance [39]. However, the reliability of the NFT effect is still not conclusive. Although Bauer [5] found increments in alpha band after NFT, several studies $[16,40]$ reported that changes on the spectral content of the EEG were not always present. Furthermore, it was suggested that significant changes in the EEG due to NFT depends on the training protocol [16].

There are several studies focused on neural changes due to NFT [6, 23, 39, 40]. Although it is an unusual method, learning control of brain rhythms using a BCI system has also been studied [33]. Similarly, there are also studies that focus on neuropsychological changes due to NFT in elderly people $[1,36,41]$. Elderly people undergo numerous changes that imply poorer cognitive performance than young adults $[6,19]$. For instance, visuospatial perception or 
memory and attention are cognitive functions that seem to be affected in the elderly [12]. Despite of the fact that some older adults perform cognitive tasks as well as young people, normal aging implies changes on neural features and brain plasticity which is one of the most prominent concerns in the elderly [19]. Furthermore, according to the United Nations [48], one in every 3 persons in developed countries will be 60 years or older in 2050. Therefore, it seems clear that the study of applications aimed at helping the elderly is of paramount importance. Although it is common to find changes in the EEG using different NFT protocols, there is controversy about effects on cognitive performance or the transference of the feedback beyond the training sessions. Not all the studies obtain cognitive effects due to NFT [36]. It is also common to find an increase in cognitive processing speed and executive functions but not in memory [1]. However, there are also studies that found an increase of memory when users trained theta band [41]. Therefore, further analyses on the effects of NFT in the elderly are needed.

In our study, we apply NFT with MI-BCI to study the potential benefits in elderly people. For this purpose, a MI-BCI application was developed, designed and assessed in the present work. Traditional NFT methodology provides feedback by means of LED lights or audio tones of a specific biofeedback apparatus [28, 29], changes in a display as the saturation of a colored square [47] or changing the height of a colored bar [46]. We hypothesized that the proposed method (MI-BCI) could be a possible alternative to the traditional NFT approach, avoiding the use of conventional apparatus to provide feedback. For this purpose, NFT effects have been studied not only observing changes in the EEG spectrum by means of relative power (RP) measures, but also through the study of changes in different cognitive functions by means of Luria Adult Neuropsychological Diagnosis (Luria-AND). Thus, the aim of this study is to compare the traditional NFT approach to the proposed MI-BCI method.

\section{Methods}

\subsection{Participants}

A total of 63 subjects were recruited by the 'Centro de Referencia Estatal de San Andrés del Rabanedo' (CRE) through advertisements and informative meetings with elderly people. All subjects were older than 60 years, healthy, and with similar educational level. None of them had previous BCI experience (BCI-naives). Population was randomly divided (taking into account age and gender) into a control group (32 subjects) and a NFT group (31 subjects)

The control group was composed of 23 females and 9 males (mean age $=68.0 \pm 5.6$ years, range $=60-80$ ), while the NFT group consisted of 18 females and 13 males (mean age $=68.3 \pm$ 4.3 years, range $=60-81)$. Nonsignificant differences were observed in the mean age or gender ( $p>0.05$, Mann-Whitney $U$-test) between both groups. Participants were recruited in three stages: (i) first stage (November-December 2012), 20 participants (10 controls and 10 NFT 
subjects); (ii) second stage (January-February 2013), 20 participants (10 controls and 10 NFT subjects); and (iii) third stage (May-June 2013), a total of 23 participants (12 controls and 11 NFT subjects. The first and the second stages served for assessing changes in several cognitive functions. Then, a new population was recruited in order to consolidate these findings by means of the analysis of spectral changes in the EEG. Thus, the protocol was redesigned just to include the recording of EEG in resting state conditions before and after using the MI-BCI tool. EEG changes were also assessed for reinforcing cognitive changes obtained during the previous stages. In summary, during the two first stages, we consider only cognitive changes assessed by means of Luria-AND tests. Thus, EEG was not recorded during training sessions. In the third stage, cognitive changes were reinforced with EEG spectral changes measured in additional subjects.

All participants (control and NFT groups) were free of psychotropic medication (nicotine not included) and without previous history of psychiatric or neurological disorders or substance abuse. The study was approved by the local ethics committee (University of Leon, Spain). All subjects gave their informed consent for participation in the study.

\subsection{EEG recordings}

Sensorimotor rhythms are activated in central brain region [22, 45]. Hence, EEG was recorded using 8 active electrodes $(\mathrm{F} 3, \mathrm{~F} 4, \mathrm{~T} 7, \mathrm{C} 3, \mathrm{Cz}, \mathrm{C} 4, \mathrm{~T} 8$, and Pz) placed in an elastic cap according to the international 10-20 system [25]. Data were referenced to a common reference placed in the ear lobe. The ground electrode was located at AFz channel. Signals were amplified by a g.USBamp amplifier (Guger Technologies OG, Graz, Austria) and digitally stored at a sampling rate of $256 \mathrm{~Hz}$.

During NFT tasks, the EEG signals were processed in real time using the BCI2000 generalpurpose system [35] for the NFT group. EEG data were filtered online with a bandpass filter between 0.1-60 Hz. Furthermore, additional recordings during a 2-min eyes closed resting-state condition were acquired from the NFT group recruited in the third stage. These recordings were obtained at the beginning and the end of the NFT protocol in order to assess EEG changes due to the five sessions of neurofeedback. Thus, potential cognitive changes in the two first stages could be reinforced by spectral changes of the EEG recordings.

\subsection{Design of the experiment}

The experiment consisted of four steps:

- Step 1 (pre-scores): control and NFT groups performed a neurological test called LuriaAND test [8]. This pre-test serves as the reference to assess neuropsychological characteristics of all subjects before the NFT. 
- Step 2: NFT group performed NFT tasks during five sessions based on motor imagery strategies using a BCI system. At the beginning of the first session and at the end of the last session, 2-min EEG with eyes closed on resting-state conditions were recorded for offline analysis from 11 subjects (last stage of the NFT group). In the present study, our novel MI$\mathrm{BCI}$ tool was based on controlling the cursor presented in a screen. Users were guided to imagine hand movements to lead the cursor to the correct target. This application and the implemented task are described in the 2.5 subsection.

- Step 3 (post-scores): all subjects performed the Luria-AND test again in order to assess potential changes in different neuropsychological functions.

- Step 4: an offline analysis was carried out to evaluate the influence of NFT in the power spectrum of the EEG and the Luria-AND scores.

\subsection{Luria Adult Neuropsychological Diagnosis}

Luria-AND test includes nine subtests distributed among five different brain functions: visuospatial (visual perception and spatial orientation), oral language (receptive speech and expressive speech), memory (immediate memory and logical memory), intellectual (thematic draws and conceptual activity), and attention (attentional control) [8]. Control and NFT groups performed the Luria-AND test twice: at the beginning (pre-scores) and at the end (post-scores) of the study. Thereby, it is possible to investigate changes due to NFT tasks in these five cognitive functions under study.

\subsection{Neurofeedback training protocol by brain computer interface system}

NFT was designed for training motor imagery that implies ERS/ERD of alpha and beta frequency bands in the EEG. In order to provide feedback to the users, spatial nearest-neighbors laplacian over C3, Cz, and C4 was applied. Spectral bands of $3 \mathrm{~Hz}$ centered on 12, 18, and 21 $\mathrm{Hz}$ were used. Since alpha power suppression is positively correlated with cognitive performance [33] and beta band activity is associated with different cognitive and motor functions [3], training these spectral bands could modify neurocognitive functions.

NFT experiment consisted of five sessions (once a week). In each session, participants were encouraged to perform five different tasks starting with the easiest activities. The difficulty was increased in the following sessions.

It is important to clarify that the training was not performed in the traditional way. Usually, NFT protocols use commercial NFT apparatus [28, 29, 46]. However, in this work, a MI-BCI system was used for feedback, resulting in an interactive application. It was not necessary to use a conventional NFT apparatus. In this approach, we used: (i) EEG recording system; (ii) laptop to process the EEG in real time; (iii) the developed MI-BCI system installed in the laptop and; 
(iv) an additional display to provide feedback to the user. The feedback was provided by means of the movement of an item in a screen, which is controlled by motor imagery tasks.

The new application was developed in C++ language as an extension of the BCI2000 system [35], using Microsoft ${ }^{\circledR}$ Visual Studio 2010. The application was installed in a laptop with an Intel Core i7-2600@ $2.30 \mathrm{GHz}$ processor and 8 GB RAM with Windows 7 operating system installed.

The five types of NFT activities, performed by the MI-BCI system, are described below (figure 1):

- T1. The first task consists of learning to imagine hand movements. Hence, the proposed interface is composed of two types of exercises: imagining right hand movements when a closed door is displayed on the screen and the similar procedure with the left one when a

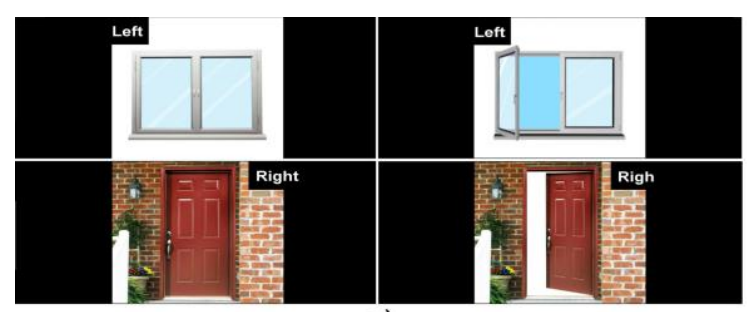

a)

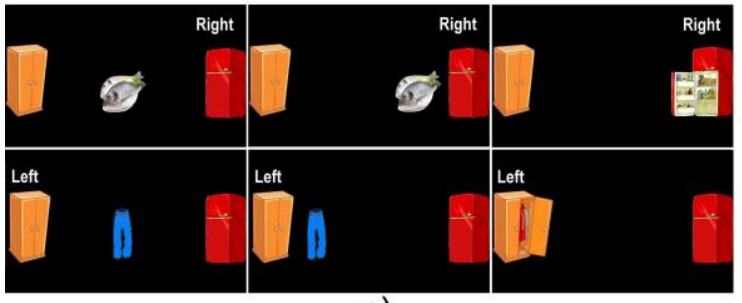

c)

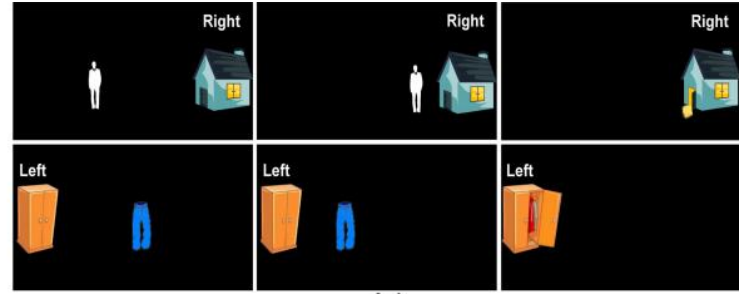

b)

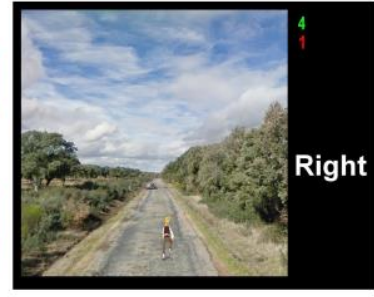

d)
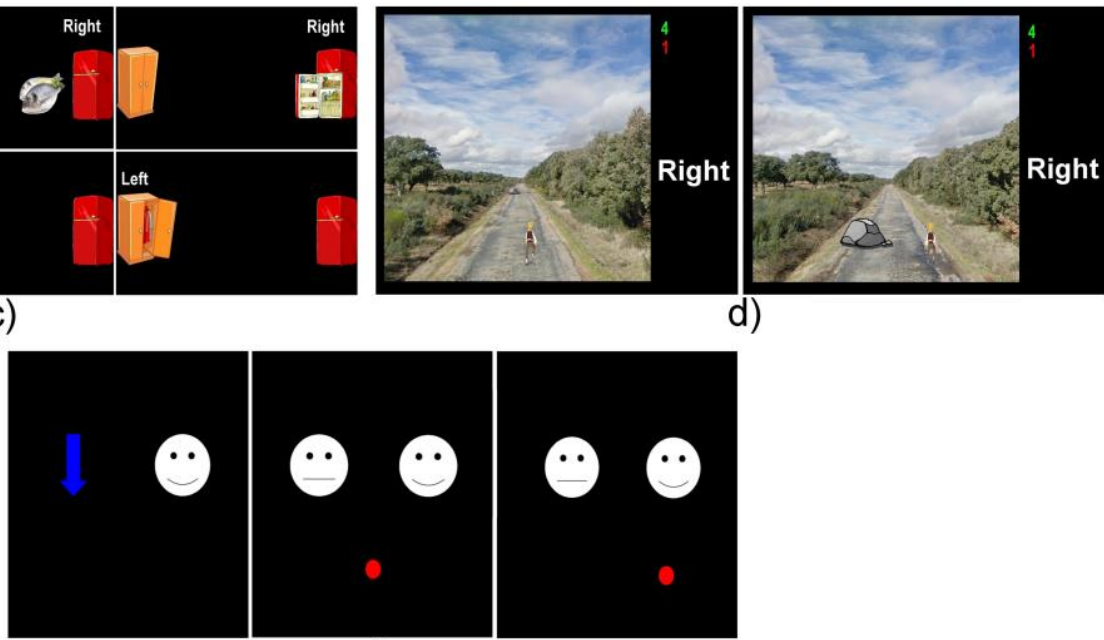

e)

Fig. 1 Screenshots of the five NFT tasks: a) T1 during motor imagery of left hand (top) and the right hand (bottom); b) T2 during motor imagery of the right hand (top) and the left hand (bottom); c) T3 during motor imagery of the right hand (top) and the left hand (bottom); d) T4 during two different moments of the task where the user must overcome an obstacle; and e) T5 during three different moments of the task, where the user must remember the repeated item and reach the correct side moving the red ball. The MI-BCI system provides feedback opening the door or window (T1), or moving the cursor (T2, T3, T4 and T5), when spectral changes in the trained frequency bands in contralateral sensorimotor areas are detected 
closed window is displayed instead (figure 1a). The visual feedback (open or not the door or window) allows the user to know when he/she is executing the proposed task suitably so that he/she can keep or change the strategy.

- T2. The second task is aimed at moving a cursor in horizontal direction in order to reach a target randomly located on the right or the left of the screen. The user has to imagine hand movements again and the cursor moves continuously over the screen. This continuous feedback allows the user to keep or change the motor imagery strategy. The cursor and targets are represented by different pairs of related pictures: person-house, fish-fridge, and trousers-wardrobe (figure 1b).

- T3. The task increases the difficulty by showing two possible targets on the screen: a right one (a picture related to the cursor) and a wrong one (unrelated to the cursor) (figure 1c). Users have to move the cursor towards the correct target by means of motor imagery.

- T4. In this task, a path crossing a park is shown to the user. The screen displays a person walking forward continuously. The user is able to control the horizontal movement, by means of hand motor imagery strategies, overcoming different obstacles that appear across the path: puddles, trees, animals, etc. (figure 1d).

- T5: This exercise combines hand motor imagery tasks with memory exercises. Firstly, two images are displayed on the screen during three seconds. Then, they disappear and two images are newly shown at the right and left of the screen: only one matches some of the initial images. The user has to identify what of these images appeared at the beginning of the trial and move the cursor towards it (figure 1e).

Figure 2 shows the equipment necessary to carry out the NFT in the approach presented in this work. In addition, figure 2 also show a real user performing the training (T4).

The two first sessions contain several trials of $\mathrm{T} 1$ in order to learn and practice motor imagery strategies, as figure 3 shows. The next tasks consisted of logical relation (T2, T3, and T4) and memory exercises (T5) that have to be solved by means of the movement of a 1D cursor. This increases the intra- and inter-session complexity of the training without increment excessively the duration of each session, which leads to an increase of training efficiency. Duration of each session was approximately 90 minutes, depending on the inter-trial and intertask and pauses. Thus, each trial requires the following durations to perform it:

- Each trial of T1 task requires a fixed time of 8 seconds.

- Each trial of T2 and T3 tasks requires a maximum of 18 seconds.

- Each trial of T4 task requires a fixed time of 24 seconds.

- Each trial of T5 task requires a maximum of 17.7 seconds. 


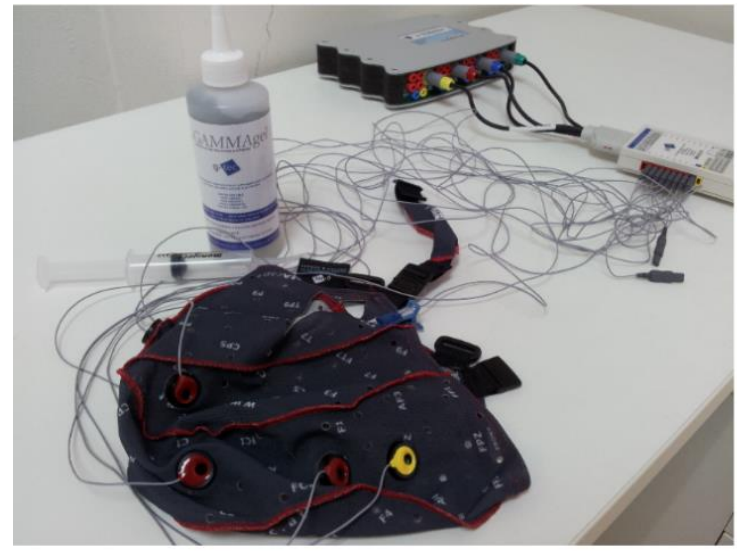

a)

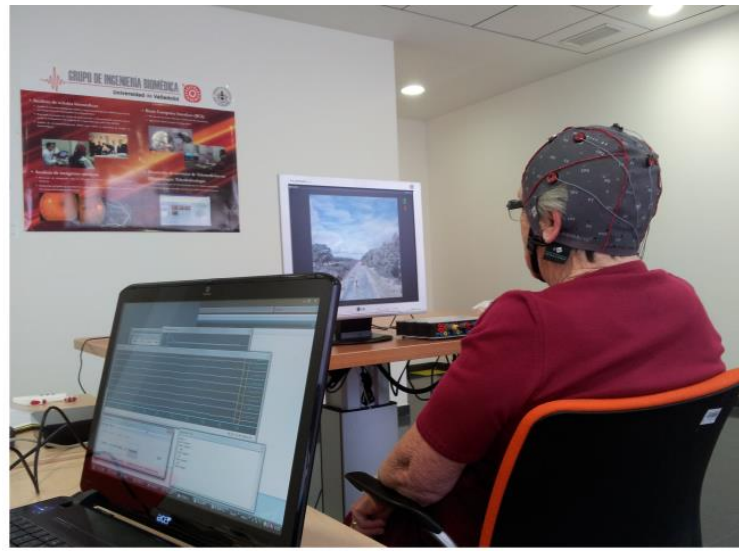

b)

Fig. 2 a) EEG recording equipment (Guger Technologies OG, Graz, Austria): the cap with 8 active electrodes, the amplifier and the gel for reduce the impedance; b) a real end-user performing the training (T4), the EEG recording equipment, a laptop with the MI-BCI system installed for processing the signals in real time and a display for providing feedback

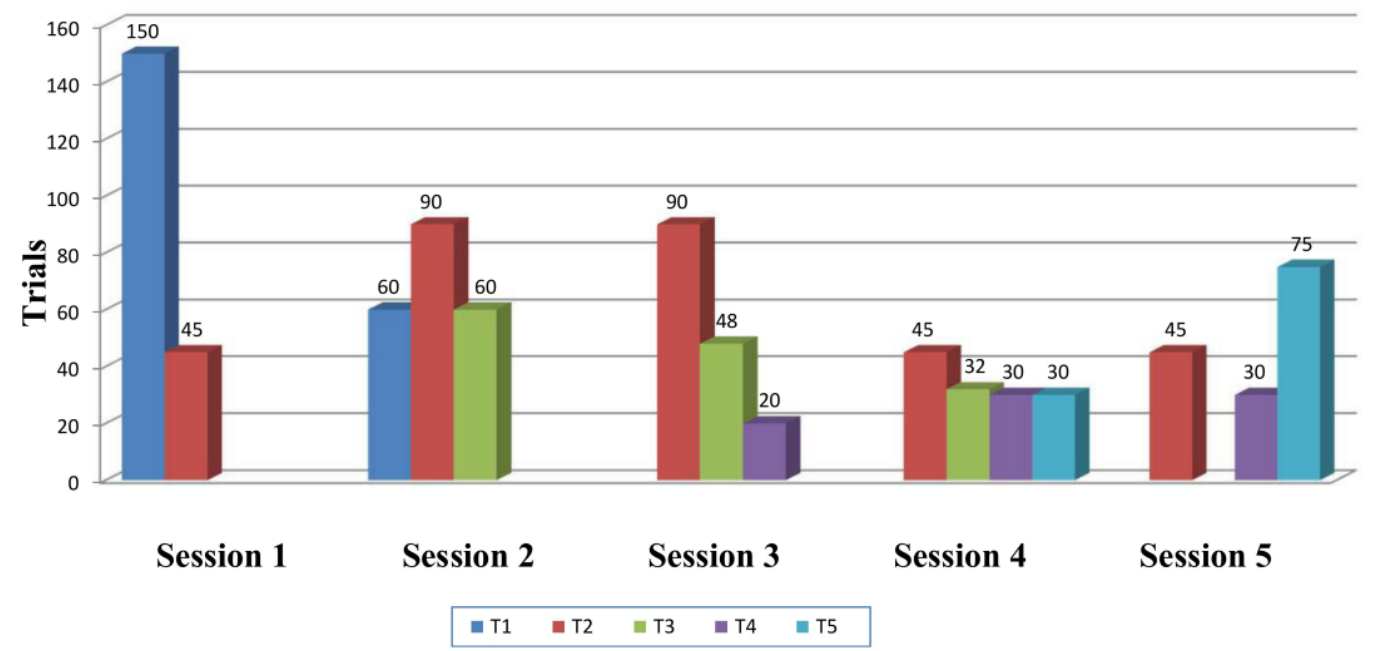

Fig. 3 Experimental design of each session (once per week). Complexity is increased across sessions by means of including more logical relationships and memory tasks. The number of trials of each type is shown

The basis of all these NFT tasks is motor imagery strategy presented by the developed MIBCI tool. Therefore, the training is based on voluntary modulation of EEG around 12, 18, and $21 \mathrm{~Hz}$. These frequencies are used to provide the feedback to the user. Brain activity of these mental work focuses on the area of centroparietal brain [32]. However, working memory is associated with frontal cortex [9] and posterior areas of the brain (for the maintenance function), including the parietal cortex [11]. Hence, several regions of the brain are trained modifying the plasticity of different cognitive functions. 


\subsection{Offline data processing}

\subsubsection{Luria analysis}

Luria-AND scores were analyzed in two ways. First, subtests of the nine cognitive features were analyzed separately. The nine cognitive features are: visual perception, spatial orientation, receptive speech, expressive speech, immediate memory, logical memory, thematic draws, conceptual activity, and attentional control. This feature scores were provided by the LuriaAND test [8]. Secondly, statistical analyses by means of Wilcoxon and Mann-Whitney tests (see statistical analysis section for details) provide independent measures of the potential changes of each subtest. On the other hand, a global analysis was carried out. Therefore, by means of a normalization of the test scores, data can be clustered into a single neurocognitive value (SNV) for all tests. Then, the neurocognitive scores of SNV for control and NFT groups were compared.

\subsubsection{EEG analysis}

EEG data were processed using Matlab R2011b, MathWorks Inc, USA, by means of custom scripts and the available functions on Statistics and Machine Learning Toolbox and Signal Processing Toolbox. Ocular artefact correction was carried out by means of independent component analysis (ICA) [24]. Next, for each channel, the $R P$ for the $3 \mathrm{~Hz}$ bands centered at 12,18 , and $21 \mathrm{~Hz}$ was computed for the 2-min rest measurements for the NFT group recruited in the third stage. $R P$ is defined as the ratio of the area enclosed under the power spectral density (PSD) in the frequency band of interest to the total area under the PSD:

$$
R P_{f_{c}}=\frac{\sum_{f \in B} \operatorname{PSD}(f)}{\sum_{f=0.1 \mathrm{~Hz}}^{60 \mathrm{~Hz}} \operatorname{PSD}(f)}, \quad B \in\left[f_{c}-1.5 \mathrm{~Hz}, f_{c}+1.5 \mathrm{~Hz},\right] \text { with } f_{c}=12,18,21 \mathrm{~Hz}
$$

For this purpose, the PSD was previously computed using the nonparametric Welch method, which is suitable for the analysis of nonstationary signals [43]. Hamming window of $2^{13}$ samples (32 seconds), along with a $90 \%$ overlap and FFT of $2^{13}$ points was used. This epoch length is long enough to collect several signal periods of alpha or beta frequency bands taken into account the sampling rate of $256 \mathrm{~Hz}$. Then, each PSD was normalized dividing the amplitude by its total power. Finally, the ERD/ERS were analyzed by means of Mann-Whitney $U$-test. 


\subsubsection{Statistical analysis}

Concerning Luria-AND tests, descriptive analysis was carried out in order to explore the distribution of the pre and post-scores. First, the Kolmogorov-Smirnov test and the Levene test were applied to evaluate the normality and the homoscedasticity of the distributions, respectively. After we observed that the pre and post-scores did not meet the parametric assumptions, nonparametric tests were used in order to evaluate our results. Scores of these tests were analyzed in two ways: (i) nonparametric Mann-Whitney $U$-test (statistical significance $p<0.01)$ was used to assess the statistical differences in the scores of each neuropsychological feature between both groups (intergroup analysis) and (ii) nonparametric Wilcoxon signed-rank test $(p<0.01)$ was applied in order to assess the statistical differences between the scores of pre and post-tests (intragroup analysis). Hence, a total of five $p$-values were calculated for each cognitive feature:

- Comparison of Luria-AND pre-scores between control and NFT group using MannWhitney test.

- Comparison of post-scores between control and NFT group using Mann-Whitney test.

- Comparison of the differences $(\Delta)$ in Luria-AND pre-scores and post-scores between control and NFT groups using Mann-Whitney test.

- Comparison of the pre and post-scores in the control group using Wilcoxon test.

- Comparison of the pre and post-scores in the NFT group using Wilcoxon test.

Regarding EEG data, changes in the $3 \mathrm{~Hz}$ bands centered at 12, 18, and $21 \mathrm{~Hz}$ were assessed. Since intragroup data did not meet parametric assumptions, $R P$ values at these frequency bands were compared using boxplots and Wilcoxon statistical test.

\section{Results}

\subsection{Luria results}

The main findings of the analysis of Luria-AND test results are summarized in table 1 . It shows several aspects that are important to note:

- The analysis of intergroup pre-scores suggests that both groups (NFT and control groups) presented similar distribution for each neuropsychological feature: there were no significant differences $(p<0.01)$ between control and NFT groups before starting the NFT protocol.

- In regard to the intergroup post-scores, there are significant differences in the score distribution between both groups for three features: visual perception $(p=0.00017)$, immediate memory $(p=0.00058)$ and conceptual activity $(p=0.00651)$.

- Changes $(\Delta)$ in pre- and post-scores between both groups were assessed and four out of them were significant: visual perception $(p=0.00013)$, receptive speech $(p=0.00288)$, immediate memory $(p=0.00005)$, and thematic draws $(p=0.00260)$. 
Table 1. Statistics associated to Mann-Whitney $U$-test (intergroup analysis) and Wilcoxon signed-rank test (intragroup analysis) for the scores of the Luria tests for each neuropsicological feature. The significant values $(p$ value $<0.01$ ) have been highlighted.

\begin{tabular}{|c|c|c|c|c|c|c|}
\hline $\begin{array}{l}\text { Neuropsychological } \\
\text { function }\end{array}$ & Feature & $\begin{array}{l}\text { NFT group vs. } \\
\text { Control group } \\
\text { (Pre vs. Pre) }\end{array}$ & $\begin{array}{l}\text { NFT group vs. } \\
\text { Control group } \\
\text { (Post vs. Post) }\end{array}$ & $\begin{array}{l}\Delta \text { Control group } \\
\text { vs. } \Delta \text { NFT group }\end{array}$ & $\begin{array}{l}\Delta \text { Control group } \\
\text { (Pre vs. Post) }\end{array}$ & $\begin{array}{l}\Delta \text { NFT group } \\
\text { (Pre vs. Post) }\end{array}$ \\
\hline Visuospatial & Visual perception & 0.34578 & 0.00017 & 0.00013 & 0.04241 & 0.00000 \\
\hline \multirow{2}{*}{ Oral language } & Receptive speech & 0.63202 & 0.12163 & 0.00288 & 0.10427 & 0.00000 \\
\hline & Expressive speech & 0.24178 & 0.01025 & 0.02275 & 0.07707 & 0.00016 \\
\hline \multirow{2}{*}{ Memory } & Immediate memory & 0.18705 & 0.00058 & 0.00005 & 0.36352 & 0.00000 \\
\hline & Logical memory & 0.74545 & 0.25419 & 0.06112 & 0.04240 & 0.00068 \\
\hline \multirow{2}{*}{ Intellectual } & Thematic draws & 0.21621 & 0.01661 & 0.00260 & 0.65469 & 0.00001 \\
\hline & Conceptual activity & 0.05581 & 0.00651 & 0.07305 & 0.06688 & 0.00015 \\
\hline Attention & Attentional control & 0.36600 & 0.16619 & 0.98565 & 0.13780 & 0.13714 \\
\hline
\end{tabular}

- About intragroup analysis, no statistical significant differences between pre and post LuriaAND scores were found for the control group.

- Increments in all cognitive features, except for attentional control, were significant for the NFT group $(p<0.01)$.

In order to facilitate the perception of the changes in Luria-AND scores, the average among all subjects of pre and post-scores for both groups are shown in figure 4 . While scores for the control group almost remained unchanged, scores for the NFT group increased after performing the NFT protocol.

With the purpose of measuring global changes in all neuropsychological functions, scores of all Luria-AND tests were normalized between 0 and 1 . Then, average of the scores of all cognitive functions was calculated obtaining the $S N V$. Histograms were constructed from these values for the post-test in the control and the NFT groups, which are shown in figure 5. Then, the corresponding curve approximation for each histogram was estimated by means of polynomial fitting with least squares error of fifth order. While the median of the histogram for the control group did not almost shift to larger scores (from 0.598 to 0.615 ), the median for the NFT group moved rightward from 0.628 to 0.706 , reflecting the global increase in the neurocognitive scores.

\subsection{EEG results}

EEG signals were processed in order to obtain $R P$ in the $3 \mathrm{~Hz}$ bands centered at 12,18 , and $21 \mathrm{~Hz}$. Thus, $264 R P$ values were obtained for the pre-NFT (11 subjects $\times 8$ channels $\times 3$ spectral bands) and the same for the post-NFT. Figure 6 shows the differences in $R P$ between the pre-NFT and post-NFT. When the difference is positive, it means that $R P$ in the post-NFT for that channel is larger than $R P$ in the pre-NFT. All differences were positive in this study. Thus, $R P$ increased for each of the channels. Particularly, channels marked with one asterisk (*) imply $p<0.05$, while two asterisks $(* *)$ mean $p<0.01$. In general, frontal and temporal regions showed the largest increases. Furthermore, the $R P$ average from each channel of all participants in NFT-session was compared with the same measure in the post-NFT for 12, 18, and $21 \mathrm{~Hz}$. 


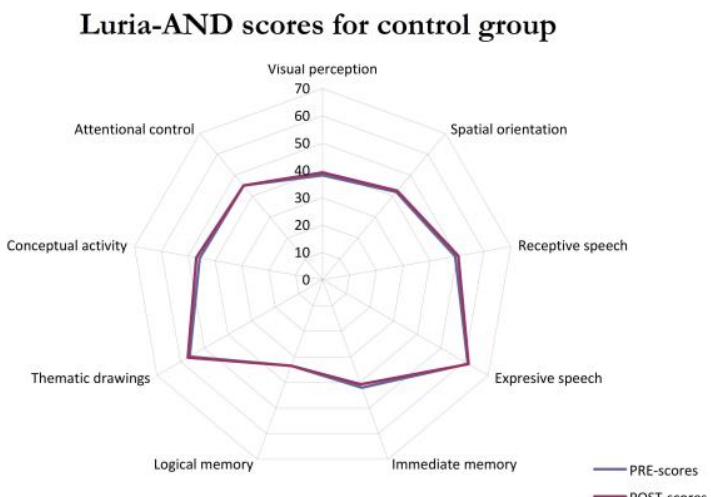

a)

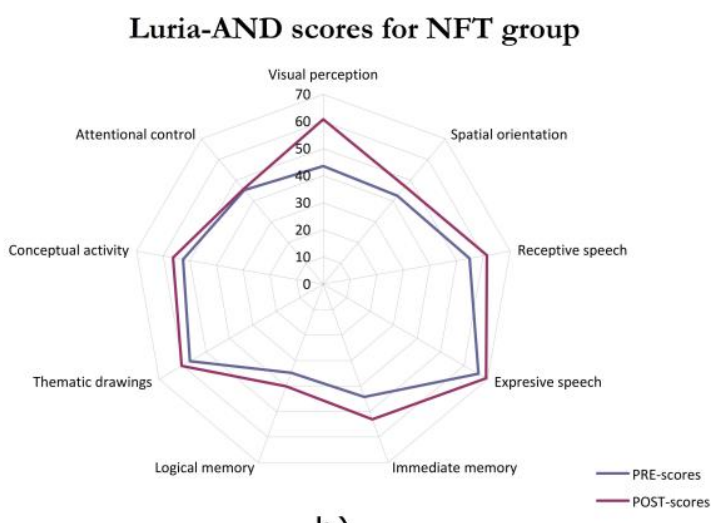

b)

Fig. 4 Radial graphics for the control group (a) and the NFT group (b) of the scores of LuriaAND test. Scores for the control group did not change, while scores of all neurocognitive functions increased after NFT for the other group

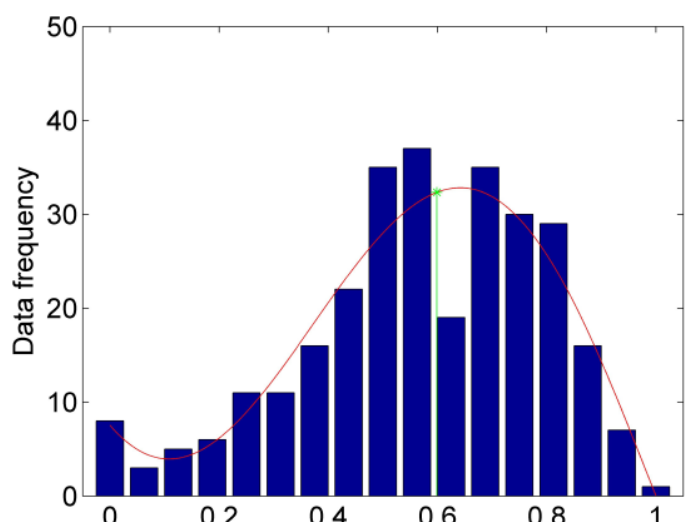

Normalized scores from control group in the pre-test

a)

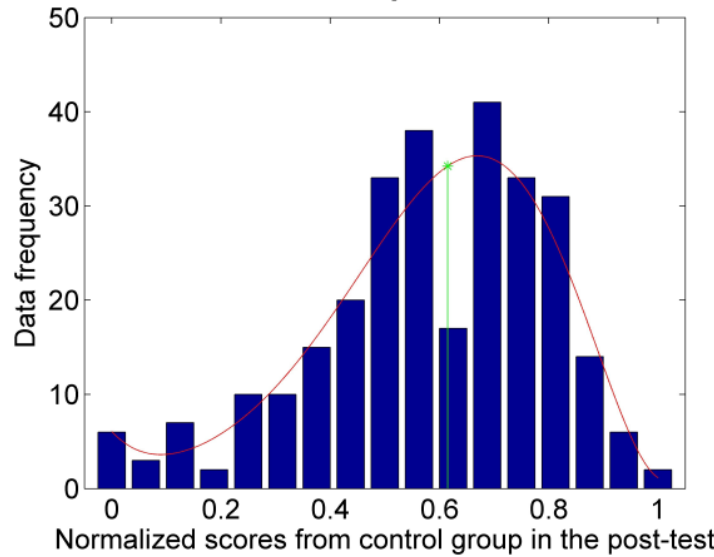

c)

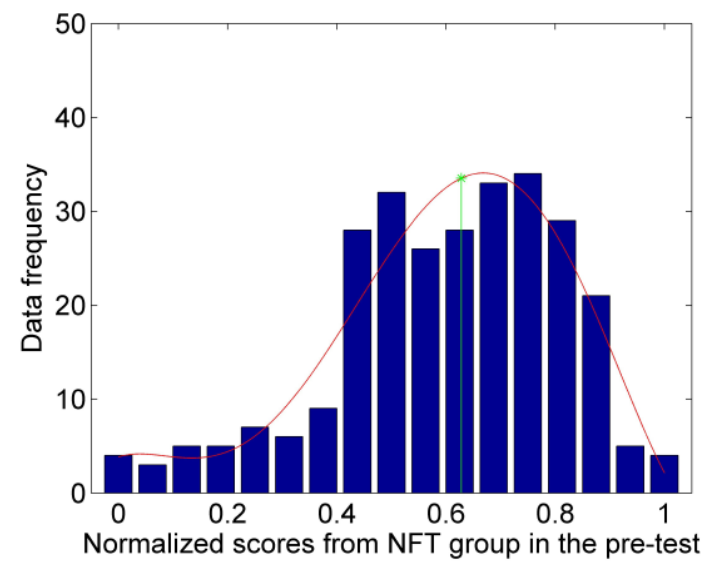

b)

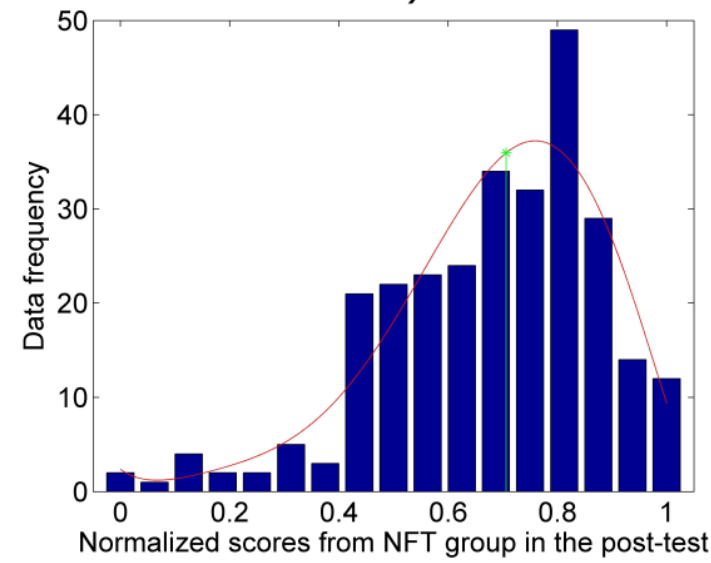

d)

Fig. $5 S N V$ for the control group in the pre-test (a), for NFT group in pre-test (b), for control group in the post-test (c) and for NFT group in the post-test (d). Scores were increased (moved rightward) when users performed the NFT protocol 
These changes were significant for frequency bands centered in $18 \mathrm{~Hz}(p=0.00062)$ and in 21 $\mathrm{Hz}(p=0.00186)$, but not for the band centered in $12 \mathrm{~Hz}(p=0.23450)$. However, five out of the eight channels achieved significant differences in the intrachannel comparison for frequency band centered in $12 \mathrm{~Hz}$, as it is shown in figure 6 .

\section{Discussion}

In the present study, a motor imagery-based BCI was used as a strategy to slow down the effect of aging. To achieve this goal, we implemented a MI-BCI application to carry out NFT tasks. Methodology assessment was performed looking for significant differences in Luria-AND test and $R P$. We observed that, regarding intergroup comparisons in table 1, no significant differences were found between the control group and the NFT group of Luria-AND values during the pre-test. It indicates that the score distributions of the groups are similar before NFT. This finding is relevant because it provides greater value to intergroup comparisons after training. In this regard, the intergroup evaluation of post-scores together with the comparison of the increments between pre and post scores reveals significant differences in most of the cognitive functions after performing NFT. With regard to intragroup scores, our results show that there were not significant changes for any of the cognitive functions for the control group. On the other hand, in the NFT group, statistical significant differences $(p<0.01)$ were found in all cognitive functions except for the attentional control one. Hence, according to these evidences, cognitive improvements in the visuospatial, language, memory, and intellectual functions could be linked with NFT.

Previous researches focusing on the alpha frequency $(8-13 \mathrm{~Hz})$ suggest that this brain activity plays a role in intelligence and memory $[5,14,27]$. Furthermore, changes in alpha

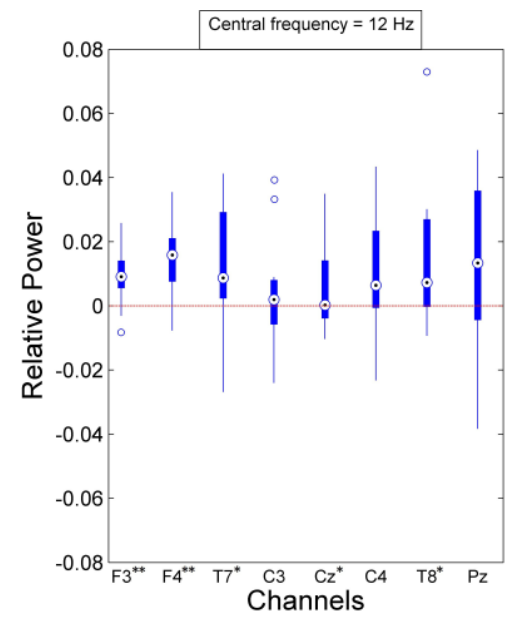

a)

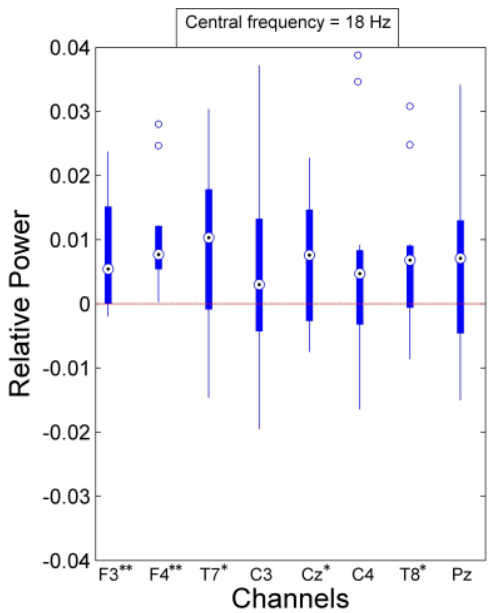

b)

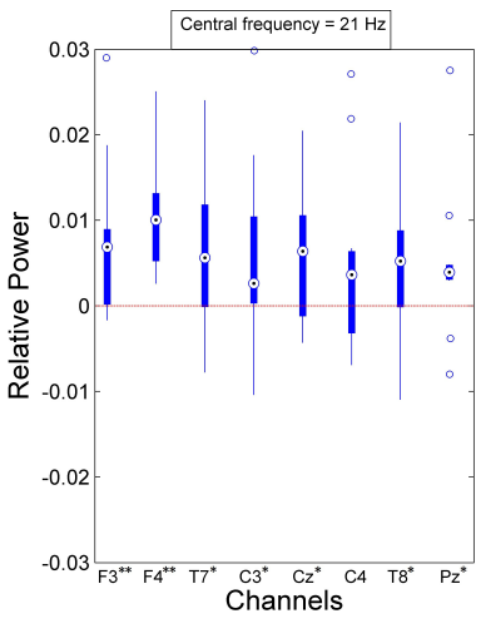

C)

Fig. 6 Boxplots of $R P$ of the differences (post-pre) for spectral band centered in $12 \mathrm{~Hz}$ (a), $18 \mathrm{~Hz}$ (b) and $21 \mathrm{~Hz}$ (c). Significant differences with $p<0.05$ are marked with one asterisk while significant differences with $p<0.01$ are marked with two asterisk 
rhythm symmetry during learning seem to be related with visuospatial cognitive function [7]. Nevertheless, Bauer did not found significant enhancement in these cognitive functions after the training of 13 participants [5]. There are several possibilities to explain the fact that NFT of alpha band do not show discernible effect on short-term memory performance [40]: the limited number of NFT sessions and the need of separating alpha into distinct components (e.g. in upper alpha). Vernon [40] suggested that lower alpha frequencies are associated with attentional demands, while the upper alpha frequency is related to semantic memory [40]. This could explain why, in the present study, improvements in Luria-AND scores for the memory and intellectual functions were found but not in attentional control, since only upper alpha was trained. Furthermore, our results suggest that a reduced number of sessions (5 NFT sessions) may be enough to appreciate cognitive changes in memory and visuospatial functions. This may be due to the use of our novel motor imagery-based $\mathrm{BCI}$ application for the performing of the NFT protocol.

Regarding beta frequency band $(13-30 \mathrm{~Hz})$, different studies reported correlation between this frequency range and cognitive functions, such as language and memory [4, 26, 42]. Specifically, these studies showed a relationship between beta $(13-18 \mathrm{~Hz})$ and memory, while language processing was related to beta frequency band in two aspects [42]: (i) semantic features of word categories and lexical-semantic and (ii) complex linguistic sub-processes, such as parsing as well as syntactic and semantic binding operations. With the aim of measuring potential changes in language and memory, we focus on the NFT protocol to train beta activity. Thus, frequency bands of $3 \mathrm{~Hz}$ centered in 18 and $21 \mathrm{~Hz}$ were included in our NFT. Results showed clear evidences of changes in both cognitive functions despite the reduced number of training sessions.

These results were reinforced with quantitative analysis of EEG changes in the trained frequency bands. In this way, it is more plausible to associate cognitive changes with NFT, since otherwise, it could be due to other external unmeasured causes [40]. In the present study, significant differences in all channels except in $\mathrm{C} 4$ were found in some of the trained frequencies. Nevertheless, regarding $\mathrm{C} 4$, most of the subjects increased their $R P$ in all frequency bands under study.

Two of the most widely used methods to stimulate certain frequencies are evoking emotions [47] or presenting emotional faces [21]. Pineda et al. [33] presented MI-BCI as an alternative, which is relatively simple to perform by participants. They develop a BCI tool with a visual feedback where five participants learned to control mu rhythms by means of videogames. In fact, motor imagery has shown to be useful to control sensorimotor rhythms, often with a visual feedback and sometimes with audio feedback [30]. However, to the best of our knowledge, this is the first study that used MI-BCI to evaluate neurological changes due to NFT in elderly people. BCIs offer a user-friendly design of interfaces so that participants are more motivated to 
perform the training. Furthermore, it appears to be an efficient strategy, since scores in several cognitive functions and the spectral power in the corresponding trained frequency bands showed statistical significant increments. These results were achieved carrying out only five BCI-based NFT sessions. Therefore, the proposed method could be an alternative to traditional NFT approaches.

The present study has some limitations that must be noted. Firstly, it would be desirable to extend the population under study for statistical reasons, in particular population which performs 2-min recordings with eyes closed on resting-state conditions. Therefore, additional studies are needed to support such evidences. Nevertheless, the total population (63 participants) is larger than similar studies $[1,33]$. The second limitation is the study of the possible long-term effects of the training. A follow up of the cognitive improvements is necessary, especially due to the specific population involved in this study (elderly people). One could ascertain whether our NFT protocol served as enforcement for the elderly brain fitness or just a temporary improvement was found. Thus, a follow up of the population involved in the study is necessary in order to assess the long term cognitive improvements. Future works may be focused to resolve this lack for the particular population of elderly people.

\section{Conclusions}

This study presents promising results about the usefulness of BCI-based NFT to increase four neuropsychological features that could help in the enhancement of the brain plasticity, which is one of the most the prominent concerns in the elderly [19]. A software application controlled by means of motor imagery-based BCI allows interactive and attractive training, obtaining significant EEG and cognitive changes after only five sessions. According to the results, MI-BCI may improve four cognitive functions in elderly people, such as visuospatial, language, memory, and intellectual. This study enforces previous findings, while it opens the possibility of designing new NFT based on motor imagery strategies.

\section{Acknowledgments}

This research was supported in part by the 'Proyecto Cero' 2011 on Ageing from Fundación General CSIC, Obra Social La Caixa and CSIC, the projects TEC2014-53196 of 'Ministerio de Economía y Competitividad' and FEDER, and the project VA059U13 of "Conserjería de Educación". Finally, J. Gomez-Pilar were in receipt of a PIF-UVA grant from University of Valladolid. 


\section{References}

[1] Angelakis E, Stathopoulou S, Frymiare JL, Green DL, Lubar JL, Kounios J (2007) EEG neurofeedback: a brief overview and an example of peak alpha frequency training for cognitive enhancement in the elderly. Clin Neuropsychol 21:110-129.

[2] Arns M, Heinrich H, Strehl U (2014) Evaluation of neurofeedback in ADHD: the long and winding road. Biol Psychol 95, 108-115.

[3] Baker SN (2007) Oscillatory interactions between sensorimotor cortex and the periphery. Curr Opin Neurobiol 17(6), 649-655.

[4] Bastiaansen M, Hagoort P (2006) Oscillatory neuronal dynamics during language comprehension. Prog Brain Res 159, 179-196.

[5] Bauer RH (1976) Short-term memory: EEG alpha correlates and the effect of increased alpha. Behav Biol 17(4), 425-433.

[6] Burke SN, Barnes CA (2006) Neural plasticity in the ageing brain. Nat Rev Neurosci 7, 30-40.

[7] Chartock HE, Glassman PR, Poon LW, Marsh GR (1975) Changes in alpha rhythm asymmetry during learning of verbal and visuospatial tasks. Physiol Behav 15(2), 237-239.

[8] Christensen AL (1979) A practical application of the Luria methodology. J Clin Exp Neuropsychol 1(3), 241-247.

[9] Clark L, Blackwell AD, Aron AR, Turner DC, Dowson J, Robbins TW, Sahakian BJ (2007) Association between response inhibition and working memory in adult ADHD: a link to right frontal cortex pathology? Biol Psychiatry 61(12), 1395-1401.

[10] Coben R, Linden M, Myers TE (2010) Neurofeedback for autistic spectrum disorder: a review of the literature. Appl Psychophysiol Biofeedback 35, 83-105.

[11] Curtis CE, D’Esposito M (2003). Persistent activity in the prefrontal cortex during working memory. Trends Cogn Sci 7(9), 415-423.

[12] Craik FIM, Salthouse TA (2011) Handbook of Aging and Cognition II. Psychology Press.

[13] Daly JJ, Wolpaw JR (2008) Brain-computer interfaces in neurological rehabilitation. Lancet Neurol 7(11), 1032-1043.

[14] Doppelmayr M, Klimesch W, Stadler W, Pöllhuber D, Heine C (2002) EEG alpha power and intelligence. Intelligence 30(3), 289-302.

[15] Egner T, Gruzelier JH (2001). Learned selfregulation of EEG frequency components affects attention and event-related brain potentials in humans. Neuroreport 12(18), 4155-4159.

[16] Egner T, Zech TF, Gruzelier JH (2004) The effects of neurofeedback training on the spectral topography of the electroencephalogram. Clin Neurophysiol 115(11), 2452-2460.

[17] Gevensleben H, Holl B, Albrecht B, Vogel C, Schlamp D, Kratz O, Studer P, Rothenberger A, Moll GH, Heinrich H (2009) Is neurofeedback an efficacious treatment for ADHD? A randomised controlled clinical trial. J Child Psychol Psychiatry 50(7), 780-789.

[18] Ghaziri J, Tucholka A, Larue V, Blanchette-Sylvestre M, Reyburn G, Gilbert G, Lévesque J, Beauregard M (2013) Neurofeedback training induces changes in white and gray matter. Clin EEG Neurosci 44(4), 265-272. 
[19] Grady CL, Craik FI (2000) Changes in memory processing with age. Curr Opin Neurobiol 10(2), 224-231.

[20] Guger C, Daban S, Sellers E, Holzner C, Krausz G, Carabalona R, Gramatica F, Edlinger G (2009) How many people are able to control a P300-based brain-computer interface (BCI)? Neurosci Lett 462, 94-98.

[21] Güntekin B, Basar E (2007) Emotional face expressions are differentiated with brain oscillations. Int J Psychophysiol 64, 91-100.

[22] Hadjidimitriou S, Zacharakis A, Doulgeris P, Panoulas K, Hadjileontiadis L, Panas S (2010) Sensorimotor cortical response during motion reflecting audiovisual stimulation: evidence from fractal EEG analysis. Med Biol Eng Comput 48(6), 561-572.

[23] Hanslmayr S, Staudigl T, Fellner MC (2012) Oscillatory power decreases and long-term memory: the information via desynchronization hypothesis. Front Hum Neurosci 6, 74.

[24]Hyvärinen A, Oja E (2000) Independent component analysis: algorithms and applications. Neural Networks 13(4), 411-430.

[25] Jasper HH (1958) Report of Committee on Methods of Clinical Examination in Electroencephalography. Electroenceph Clin Neurophysiol 10, 370-375.

[26] Kim JS, Chung CK (2008) Language lateralization using MEG beta frequency desynchronization during auditory oddball stimulation with one-syllable words. Neuroimage 42(4), 1499-1507.

[27] Klimesch W (1999) EEG alpha and theta oscillations reflect cognitive and memory performance: a review and analysis. Brain Res Rev 29(2), 169-195.

[28] Lubar JO, Lubar JF (1984) Electroencephalographic biofeedback of SMR and beta for treatment of attention deficit disorders in a clinical setting. Biofeedback and self-regulation, 9(1), 1-23.

[29] Lubar JF, Swartwood MO, Swartwood JN, O'Donnell PH (1995) Evaluation of the effectiveness of EEG neurofeedback training for ADHD in a clinical setting as measured by changes in TOVA scores, behavioral ratings, and WISC-R performance. Biofeedback and self-regulation, 20(1), 83-99.

[30] McCreadie KA, Coyle DH, Prasad G (2013) Sensorimotor learning with stereo auditory feedback for a brain-computer interface. Med Biol Eng Comput 51(3), 285-293.

[31] Neuper C, Scherer R, Wriessnegger S, Pfurtscheller G (2009) Motor imagery and action observation: modulation of sensorimotor brain rhythms during mental control of a brain-computer interface. Clin Neurophysiol 120(2), 239-247.

[32] Pfurtscheller G, Neuper C (1997) Motor imagery activates primary sensorimotor area in humans. Neurosci Lett 239(2), 65-68.

[33] Pineda JA, Silverman DS, Vankov A, Hestenes J (2003) Learning to Control Brain Rhythms: Making a Brain-Computer Interface Possible. IEEE Trans Neural Syst Rehabil Eng 11(2): 181-184.

[34]Ros T, Munneke MA, Ruge D, Gruzelier JH, Rothwell JC (2010) Endogenous control of waking brain rhythms induces neuroplasticity in humans. Eur J Neurosci 31(4), 770-778.

[35] Schalk G, McFarland DJ, Hinterberger T, Birbaumer N, Wolpaw JR (2004) BCI2000: a generalpurpose brain-computer interface (BCI) system. IEEE Trans Biomed Eng 51, 1034-1043.

[36] Staufenbiel SM, Brouwer AM, Keizer AW, VanWouwe NC (2014) Effect of beta and gamma Neurofeedback on memory and intelligence in the elderly. Biol Psychol 95, 74-85. 
[37] Tan G, Thornby J, Hammond DC, Strehl U, Canady B, Arnemann K, Kaiser DA (2009) Metaanalysis of EEG biofeedback in treating epilepsy. Clin EEG Neurosci 40(3), 173- 179.

[38] Thornton KE, Carmody DP (2008) Efficacy of traumatic brain injury rehabilitation: Interventions of qEEG-guided biofeedback, computers, strategies, and medications. Appl Psychophysiol Biofeedback 33(2), 101-124.

[39] Vernon D, Egner T, Cooper N, Compton T, Neilands C, Sheri A, Gruzelier J (2003) The effect of training distinct neurofeedback protocols on aspects of cognitive performance. Int $\mathrm{J}$ Psychophysiol $47,75-85$.

[40] Vernon DJ (2005) Can neurofeedback training enhance performance? An evaluation of the evidence with implications for future research. Appl Psychophysiol Biofeedback 30(4), 347-364.

[41]Wang JR, Hsieh S (2013) Neurofeedback training improves attention and working memory performance. Clin Neurophysiol 124(12), 2406-2420.

[42] Weiss S, Mueller HM (2012) "Too many betas do not spoil the broth": the role of beta brain oscillations in language processing. Front Psychol 3, 201.

[43] Welch PD (1967) The use of fast Fourier transform for the estimation of power spectra: a method based on time averaging over short, modified periodograms. IEEE T Audio Electroacoust 15(2), 7073.

[44] Wolpaw JR, Birbaumer N, Heetderks WJ, McFarland DJ, Peckham PH, Schalk G, Donchin E, Quatrano LA, Robinson CJ, Vaughan TM (2000) Brain-computer interface technology: a review of the first international meeting. IEEE Trans Rehab Eng 8(2), 164-173.

[45] Wolpaw JR, Birbaumer N, McFarland DJ, Pfurtscheller G, Vaughan TM (2002) Brain-computer interfaces for communication and control. Clin Neurophysiol 113(6), 767-791.

[46] Young KD, Zotev V, Phillips R, Misaki M, Yuan H, Drevets WC, Bodurka J (2014) Real-time FMRI neurofeedback training of amygdala activity in patients with major depressive disorder. . PLoS ONE 9(2): e88785. doi:10.1371/journal.pone.0088785

[47]Zoefel B, Huster RJ, Herrmann CS (2011) Neurofeedback training of the upper alpha frequency band in EEG improves cognitive performance. Neuroimage 54(2), 1427-1431.

[48] United Nations, Department of Economic and Social Affairs, Population Division 2013, World Population Ageing (2013) ST/ESA/SER.A/348. Avaiable via DIALOG, http://www.un.org/en/development/desa/population/publications/pdf/ageing/WorldPopulationAgeing 2013.pdf Accessed 26 Jan 2015 

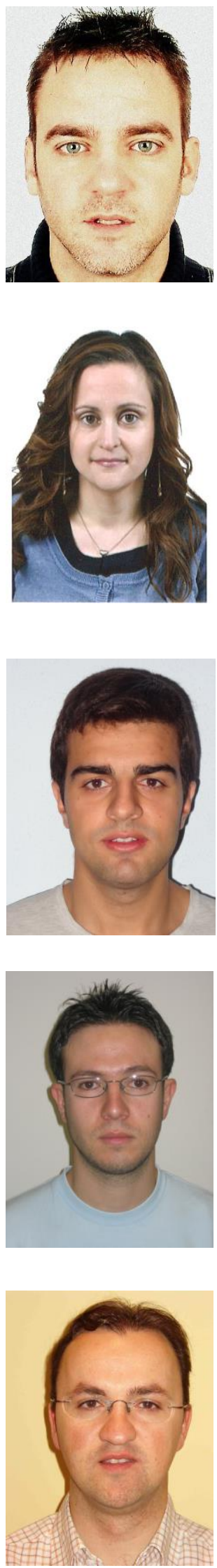

Javier Gomez-Pilar received the M.S. degree in telecommunication engineering, from the University of Valladolid, where he is currently working toward the Ph.D. degree at the Biomedical Engineering Group. His work focuses on signal processing and neurosciences.

Rebeca Corralejo received the M.S. degree in telecommunication engineering from the University of Valladolid (Spain), where she is currently working toward the Ph.D. degree.

Luis F. Nicolas-Alonso received the M.S. degree in telecommunication engineering, from the University of Valladolid. He is a member of the Biomedical Engineering Group. His current research interests include signal processing applied to braincomputer interfaces.

Daniel Alvarez received the Ph.D. degree in Telecommunication Engineering from the University of Valladolid. Since 2005, he is a member of the Biomedical Engineering Group. His work focuses on multivariate analysis and pattern recognition of biomedical signals.

Roberto Hornero is currently Professor in the Department of Signal Theory and Communications and Director of the Biomedical Engineering Group at the University of Valladolid. 